\title{
Understanding Chemical States of Copper Nanoparticles in Wood Structures by Electron Energy Loss Spectroscopy
}

\author{
Chen Wang $^{1 *}$, Alan K. Dozier ${ }^{1}$ and Pramod Kulkarni ${ }^{1}$ \\ 1. Division of Applied Research and Technology, National Institute for Occupational Safety and Health, \\ Cincinnati, OH, USA. \\ * Corresponding author: xli7@cdc.gov
}

As alternatives to the conventional ionized copper-based preservatives used in the pressure-treated lumber (PTL), micronized copper azole (MCA) compounds have become the primary sources for treating PTL products in recent years [1]. The active ingredients of MCA wood preservatives consist of basic copper carbonate (BCC) particles with sizes less than $1 \mu \mathrm{m}$ and organic co-biocide compounds [2]. The release of copper compounds has been observed during leaching in the environment and mechanical processing of the PTL products [3-5]. There is a growing concern over the potential exposure of workers to the micronized/nanoscale copper particles released during the handling and mechanical processing of copper-treated lumber. A recent toxicity study indicated that sanding of copper-treated lumber can produce nanoscale copper particles that are potentially cytotoxic [6]. However, the potential toxicity of metal nanoparticles is greatly affected by their chemical states, aspect ratios, and degree of agglomeration. In this study, we utilized electron energy loss spectroscopy (EELS) to investigate the local chemical states of various copper compounds and combined scanning transmission electron microscopy (STEM) to image the microstructures of particles.

A Taber Abraser was used to simulate the mechanical abrasion processes on the treated wood surfaces. The bulk copper-treated wood specimens were analyzed with a scanning electron microscopy (SEM) to visualize the micro-distribution of copper preservatives in the wood cell structures. Airborne particles released from the abrasion process were collected on a mixed cellulose ester (MCE) filter and processed on nickel grids for STEM and EELS analysis. The STEM images were acquired from both Bright Field (BF) and High Angle Annular Dark Field (HAADF) detectors with a probe size of 0.5-1 nm. Energy Dispersive X-ray Spectroscopy (EDS) point analysis and line scans were conducted at each region of interest (ROI) to determine the elemental composition of unknown particles. The oxidation states of copper nanoparticles were determined by the electron energy-loss near-edge structures (ELNES) of their characteristic $\mathrm{L}_{2,3}$ edges and compared with theoretical calculations.

Large particles (mostly BBC precipitates) were found accumulated in wood cell secondary layers and on membranes within the bordered pits that connect wood fibers (see Figure 1). Copper content was also identified in air samples generated by mechanical abrasion of the wood surfaces. Results of STEM and EELS analyses of wood dust are shown in Figure 2. Particles with diameters in the range from 5 to 1000 $\mathrm{nm}$ were selected and analyzed by both EDS and EELS. The ELNES of $\mathrm{CuL}_{2,3}$ edges show the distinct oxidation states of two types of free copper particles. The chemical states of the ionized copper content were also evaluated to understand the chemical transformation of copper species in wood structures [7].

\section{References:}

[1] M Freeman and C Mcintyre, Forest Products Journal 58 (2008), p.6.

[2] C Civardi, F Schwarze and P Wick, Environmental Pollution 200 (2015), p.126. 
[3] A Tegenaw et al., Environmental Science \& Technology 49 (2015), p.1294.

[4] W Platten et al., Science of the Total Environment 548 (2016), p.441.

[5] C Wang et al., Microscopy and Microanalysis. 24 (2018), p.1762.

[6] J Sisler et al., Journal of Occupational and Environmental Hygiene 15 (2018), p.311.

[7] The authors acknowledge funding from the National Institute for Occupational Safety and Health, Nanotechnology Research Center.
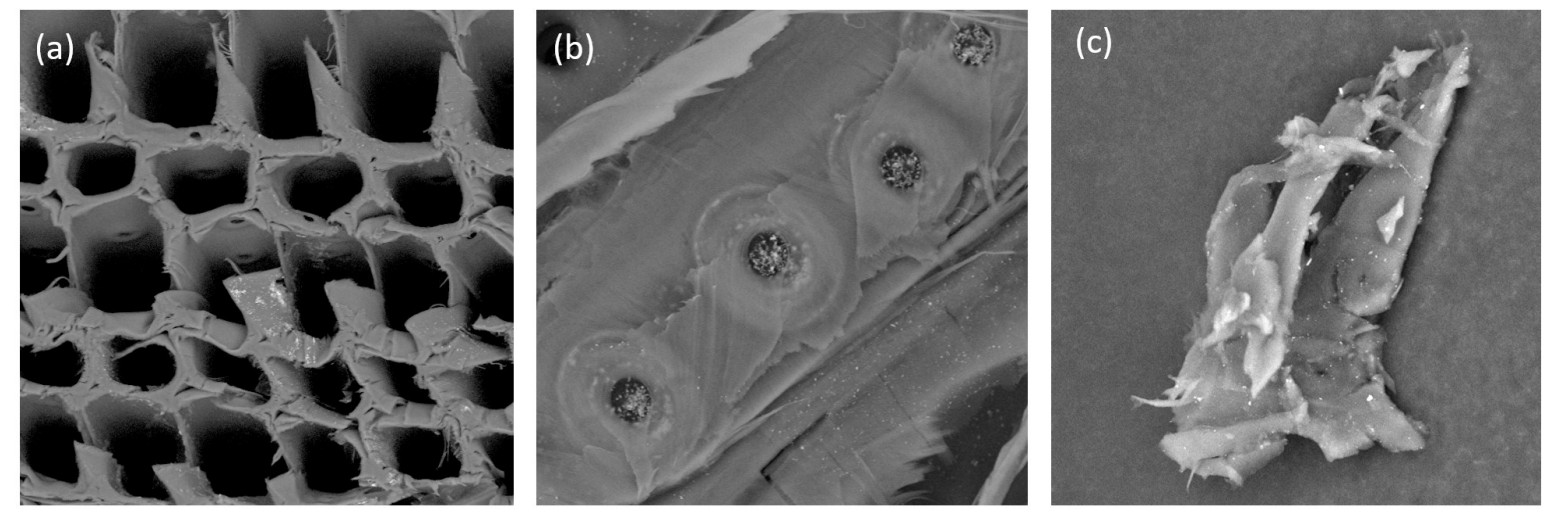

Figure 1. SEM images showing the distribution of copper-containing particles in treated wood: (a) secondary walls; (b) membranes of bordered pits; and (c) a dust particle released during the sanding processes.

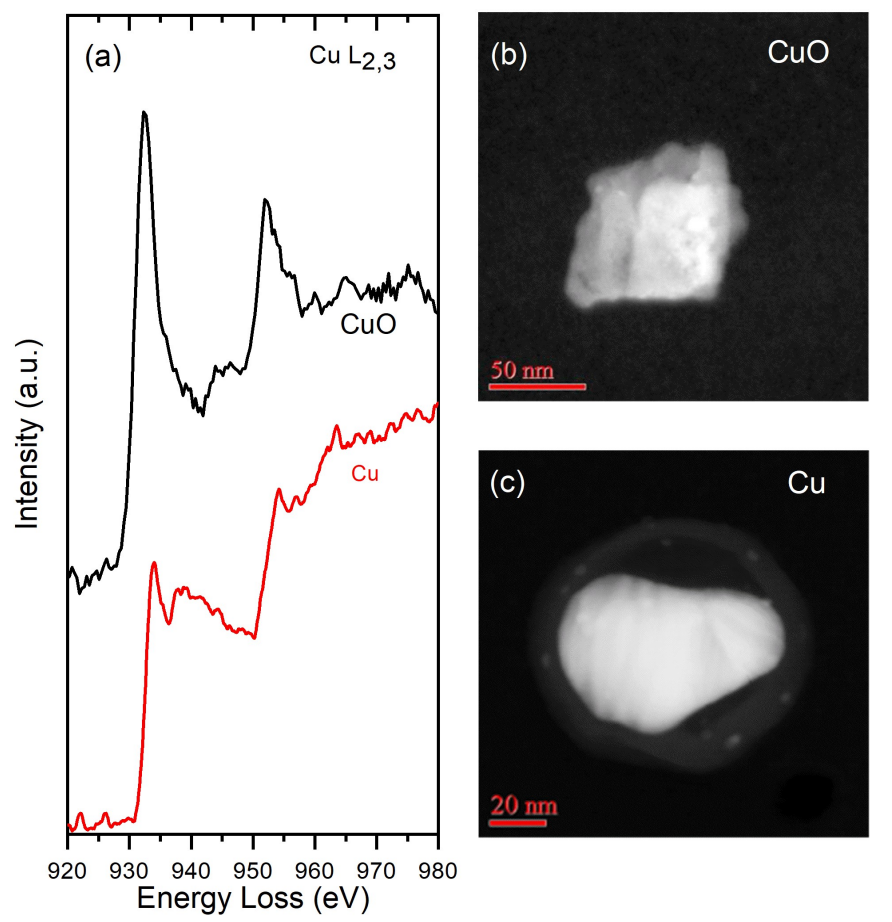

Figure 2. (a) EELS spectra showing the characteristic $\mathrm{Cu} \mathrm{L}_{2,3}$ edges of (b) $\mathrm{CuO}$ and (c) $\mathrm{Cu}$ nanoparticles. 